\title{
Electrodynamics in a Background Chiral Field
}

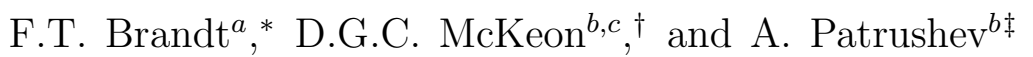 \\ ${ }^{a}$ Instituto de Física,Universidade de São Paulo, São Paulo, SP 05315-970 Brazil \\ ${ }^{b}$ Department of Applied Mathematics, University of \\ Western Ontario, London Canada N6A $5 B^{7} 7$ and \\ ${ }^{c}$ Department of Mathematics and Computer Science, \\ Algoma University, Sault St.Marie Canada P6A 2G4, Canada
}

\begin{abstract}
We consider the one loop effective action in four dimensional Euclidean space for a background chiral field coupled to a spinor field. It proves possible to find an exact expression for this action if the mass $m$ of the spinor vanishes. If $m$ does not vanish, one can make a perturbative expansion in powers of the axial field that contributes to the chiral field, while treating the contribution of the vector field exactly when it is a constant. The analogous problem in two dimensions is also discussed.
\end{abstract}

\section{INTRODUCTION}

Parity violating interactions with a spinor field yield several interesting consequences, among them an anomalous divergence in the axial current [1 13$]$ and the absence of bound states in a "Coulomb" axial potential [4, 5]. In this paper we consider the one loop effective action for a spinor field in the presence of a constant background chiral vector field. The analogous situation in which the interaction is parity conserving is well known [1, 6 - $[$ ] $]$.

\section{EFFECTIVE ACTION}

If a spinor $\psi$ is in the presence of a background vector field $V^{\mu}$ and a background axial field $A^{\mu}$ in four dimensional Euclidean space we have the Lagrangian

$$
\mathcal{L}=\psi^{\dagger}\left[\left(\not p-W_{+} P_{+}-W_{-} P_{-}\right)-m\right] \psi
$$

where $p=-i \partial$ and $W_{ \pm}=V \pm A$ are chiral fields. (The notation used is in the appendix.) The effective action is then given by the one loop expression

$$
\Gamma_{4}=\ln \operatorname{det}\left(\not p-W_{+} P_{+}-W_{-} P_{-}-m\right) \text {. }
$$

We now rewrite Eq. (2) as

$$
\Gamma_{4}=\left[\ln \operatorname{det}\left(\not p-W_{+} P_{+}-W_{-} P_{-}\right)+\ln \operatorname{det}\left(1-\frac{m}{\not p-W_{+} P_{+}-W_{-} P_{-}}\right)\right]
$$

*Electronic address: fbrandt@usp.br

${ }^{\dagger}$ Electronic address: dgmckeo2@uwo.ca

${ }^{\ddagger}$ Electronic address: apatrus@uwo.ca 
and then expand the second term in Eq. (3) so that

$$
\ln \operatorname{det}\left(1-\frac{m}{\not p-W_{+} P_{+}-W_{-} P_{-}}\right)=-\operatorname{tr} \sum_{n=1}^{\infty} \frac{1}{n}\left(\frac{m}{\not p-W_{+} P_{+}-W_{-} P_{-}}\right)^{n} .
$$

We now rewrite

$$
\begin{aligned}
\frac{1}{\not p-W_{+} P_{+}-W_{-} P_{-}} & =\frac{1}{\not p} \frac{1}{1-\frac{1}{\not p}\left(\mathscr{W}_{+} P_{+}+W_{-} P_{-}\right)} \\
& =\frac{1}{\not p} \sum_{n=0}^{\infty}\left[\frac{1}{\not p}\left(\mathscr{W}_{+} P_{+}+W_{-} P_{-}\right)\right]^{n}
\end{aligned}
$$

which by the properties of the projection operators $P_{ \pm}$becomes

$$
\begin{aligned}
& =\frac{1}{\not p} \sum_{n=0}^{\infty}\left[\left(\frac{1}{\not p} W_{+}\right)^{n} P_{+}+\left(\frac{1}{\not p} W_{-}\right)^{n} P_{-}\right] \\
& =\frac{1}{\not p-W_{+}} P_{+}+\frac{1}{\not p-W_{-}} P_{-} .
\end{aligned}
$$

Similarly, we have for the first term in Eq. (3)

$$
\begin{aligned}
\ln \operatorname{det}\left(\not p-W_{+} P_{+}-W_{-} P_{-}\right) & =\operatorname{tr}\left[\ln \not p-\sum_{n=1}^{\infty} \frac{1}{n}\left(\frac{1}{\not p} W_{+} P_{+}+\frac{1}{\not p} W_{-} P_{-}\right)^{n}\right] \\
& =\operatorname{tr}\left[\left(\ln \left(\not p-W_{+}\right)\right) P_{+}+\left(\ln \left(\not p-W_{-}\right)\right) P_{-}\right] .
\end{aligned}
$$

Together, Eqs. (3), (4), (5) and (6) show that

$$
\begin{aligned}
& \Gamma_{4}=\operatorname{tr}\left[\left(\ln \bigcap_{+}\right) P_{+}+\left(\ln \Pi_{-}\right) P_{-}-\frac{m}{1}\left(\frac{1}{\Pi_{+}} P_{+}+\frac{1}{\Pi_{-}} P_{-}\right)\right. \\
& -\frac{m^{2}}{2}\left(\frac{1}{\prod_{-}} \frac{1}{\prod_{+}} P_{+}+\frac{1}{\prod_{+}} \frac{1}{\prod_{-}} P_{-}\right) \\
& -\frac{m^{3}}{3}\left(\frac{1}{\bigcap_{+}} \frac{1}{\bigcap_{-}} \frac{1}{\bigcap_{+}} P_{+}+\frac{1}{\bigcap_{-}} \frac{1}{\bigcap_{+}} \frac{1}{\bigcap_{-}} P_{-}\right) \\
& -\ldots]
\end{aligned}
$$

where $\Pi_{ \pm} \equiv p-W_{ \pm}$.

If we now use the identity

$$
\operatorname{tr} X=\frac{1}{2} \operatorname{tr}\left[X+\gamma^{5} X \gamma^{5}\right]
$$

then we see that terms in Eq. (7) with odd powers of $m$ vanish. This reduces Eq. (7) to

$$
\Gamma_{4}=\frac{1}{2} \operatorname{tr}\left\{\left[\ln \left(\Pi_{+}^{2}\left(1-\frac{m^{2}}{\Pi_{-} \Pi_{+}}\right)\right)\right] P_{+}+\left[\ln \left(\Pi_{-}^{2}\left(1-\frac{m^{2}}{\Pi_{+} \Pi_{-}}\right)\right)\right] P_{-}\right\} .
$$


Under "charge conjugation" we find that

$$
\begin{aligned}
C^{-1} & \left(\not p-W_{+} P_{+}-W_{-} P_{-}-m\right) C \\
& =\left[\not p+W_{+} P_{-}+W_{-} P_{+}-m\right]^{T}
\end{aligned}
$$

and so Eq. (22) is symmetric under the replacement $W_{ \pm} \rightarrow-W_{\mp}$. (In ref. [9] the fact that $p^{\mu T}=-p^{\mu}$ was ignored.)

\section{EXPLICIT EVALUATION OF THE EFFECTIVE ACTION}

Evaluation of $\Gamma$ in Eq. (9) in closed form when $m^{2} \neq 0$ involves having to determine $\operatorname{tr} \ln \left(\not I_{ \pm} \not I_{\mp}-m^{2}\right)$. If $W_{ \pm} \neq W_{\mp}$ this is prohibitively difficult, even if $W_{ \pm}= \pm A$. In this case we must consider

$$
\operatorname{tr} \ln \left[(\not \not \pm \not A)(\not p \mp \not A)-m^{2}\right]=\operatorname{tr} \ln \left[\left(p^{\mu} \mp i \sigma^{\mu \nu} A^{\nu}\right)^{2}+2 A^{2} \pm i A_{, \lambda}^{\lambda}-m^{2}\right]
$$

which, though it is well suited for a perturbative expansion in powers of $A^{\mu}[10,11]$, does not lend itself to being evaluated even when $A^{\mu}$ corresponds to there being a constant field strength.

However, if $m^{2}=0$, or if Eq. (9) were expanded to some finite order in powers of $m^{2}$, then one is faced with evaluation of only $\frac{1}{2}\left(\Lambda_{+}+\Lambda_{-}\right)$where $\Lambda_{ \pm}=\operatorname{tr}\left[\ln \bigcap_{ \pm}^{2}\right] P_{ \pm}$. In refs. $\left[1,6\right.$, 7], it is shown that since $(\not p-\not V)^{2}=\left(p^{\mu}-V^{\mu}\right)^{2}-\frac{1}{2} \sigma^{\mu \nu} F^{\mu \nu}(F=\partial \wedge V)$ the gamma matrix trace occurring in $\Lambda_{ \pm}$involves

$$
\begin{aligned}
\operatorname{tr} e^{\frac{1}{2} F^{\mu \nu} \sigma^{\mu \nu} t} P_{ \pm} & =\operatorname{tr}\left\{\cosh K_{-} P_{+}+\cosh K_{+} P_{-}\right. \\
& \left.+\frac{t}{2} \sigma^{\mu \nu} F^{\mu \nu}\left(\frac{\sinh K_{-}}{K_{-}} P_{+}+\frac{\sinh K_{+}}{K_{+}} P_{-}\right)\right\} P_{ \pm} \\
& =4 \cosh K_{\mp}
\end{aligned}
$$

where $K_{ \pm}^{2}=\frac{t^{2}}{2}\left[F^{\mu \nu} F^{\mu \nu} \pm F^{\mu \nu} F^{* \mu \nu}\right]$. We thus see that the presence of the chiral projection operator $P_{ \pm}$in Eq. (9) serves to eliminate the contribution of $\cosh K_{ \pm}$as well as $\sinh K_{+}$ and $\sinh K_{-}$, leaving only $4 \cosh K_{\mp}$.

The background field strength $W_{ \pm}$in the gauge $x \cdot W_{ \pm}=0$ can be expanded in powers of the field strength $F_{ \pm}[12$ 14],

$$
W_{ \pm}^{\mu}=\sum_{n=0}^{\infty} \frac{-1}{n !(n+2)} x^{\nu} x^{\lambda_{1}} \ldots x^{\lambda_{n}} F_{ \pm}^{\mu \nu, \lambda_{1} \ldots \lambda_{n}}(0) .
$$

The first term in Eq. (13) corresponds to a constant background field as discussed in refs. [1, 6, 7]; higher contributions are dealt with in refs. [8, 15 17]. Other special background field configurations have been considered [1, 8, 18 20].

If $m^{2}=0$ and $W_{ \pm}= \pm A$, then we have a purely axial coupling and

$$
\Gamma_{A}^{(0)}=\frac{1}{2} \operatorname{tr}\left[\left(\ln (\not p-\not A)^{2}\right) P_{+}+\left(\ln (\not p+\not A)^{2}\right) P_{-}\right] .
$$


If $A^{\mu}$ is in the gauge $x \cdot A=0$ so that it is expressed in the form of Eq. (13) then gauge invariance is manifestly preserved since $A^{\mu}$ is expressed in terms of the field strength. If we then expand $\Gamma_{A}^{(0)}$ with this background field using the Schwinger expansion as in ref. [1, 21], then the three point function $\langle A A A\rangle$ vanishes. However, again computing $\langle A A A\rangle$ but with plane wave background axial fields, the three point function is consistent with the axial anomaly $1-3]$.

If $m^{2} \neq 0$ when $W_{ \pm}= \pm A$ then Eq. (9) reduces to

$$
\begin{aligned}
\Gamma_{A}=\frac{1}{2} \operatorname{tr}\{ & {\left[\ln \left((\not p+\not A)(\not p-\not A)-m^{2}\right)\right] P_{+}+\left[\ln \left((\not p-\not A)(\not p+\not A)-m^{2}\right)\right] P_{-} } \\
& \left.+\frac{1}{2}\left[\ln (\not p-\not A)^{2}-\ln (\not p+\not \not)^{2}\right] \gamma_{5}\right\} .
\end{aligned}
$$

There doesn't appear to be a way of evaluating this in closed form when even $A^{\mu}=-\frac{1}{2} F^{\mu \nu} x^{\nu}$ if $m^{2} \neq 0$, though with this background field $\langle A A A\rangle=0$. With a plane wave background field the axial anomaly can however be recovered [21] when $\langle A A A\rangle$ is computed by applying the Schwinger expansion [1] to Eq. (15).

Although it doesn't appear to be feasible to compute $\Gamma_{4}$ when there is a constant strength $\partial^{\mu} A^{\nu}-\partial^{\nu} A^{\mu}$ in Eq. (1), we can consider the case in which $\Gamma_{4}$ is restricted to being linear in the external axial field and the vector field is taken to be constant. In this case we begin by using Eq. (8) to write

$$
\Gamma_{4}=\frac{1}{2} \ln \operatorname{det}\left[\left(\not p-\not-\not A \gamma^{5}\right)^{2}-m^{2}\right] .
$$

Dropping those terms in Eq. (12) that cannot contribute to the contribution to $\Gamma_{4}$ that are linear in $A_{\mu}$, we see that upon letting $m^{2} \rightarrow-m^{2}$,

$$
\begin{aligned}
\Gamma_{4} \approx \frac{1}{2} \ln \operatorname{det}\left[(p-V)^{2}+m^{2}-\frac{1}{2} F^{\mu \nu} \sigma^{\mu \nu}+i A^{\mu, \mu} \gamma^{5}\right. \\
\left.+i \sigma^{\mu \nu}\left(2 A^{\mu} p^{\nu}+\frac{i}{2} G^{\mu \nu}-2 A^{\mu} V^{\nu}\right) \gamma^{5}\right]
\end{aligned}
$$

where $F^{\mu \nu}=\partial^{\mu} V^{\nu}-\partial^{\nu} V^{\mu}$ and $G^{\mu \nu}=\partial^{\mu} A^{\nu}-\partial^{\nu} A^{\mu}$. If we now employ operator regularization to expand $\Gamma_{4}$ in Eq. (17) to the term linear in $A_{\mu}$, we need the equations [21]

$$
\begin{aligned}
\frac{1}{2} \ln \operatorname{det}\left(H_{0}+H_{1}\right) & =-\left.\frac{1}{2} \frac{d}{d s}\right|_{0} \operatorname{tr} \frac{1}{\Gamma(s)} \int_{0}^{\infty} d t t^{s-1} e^{-\left(H_{0}+H_{1}\right) t} \\
=-\left.\frac{1}{2} \frac{d}{d s}\right|_{0} & \frac{1}{\Gamma(s)} \int_{0}^{\infty} d t t^{s-1} \operatorname{tr}\left[e^{-H_{0} t}+\frac{(-t)}{1} e^{-H_{0} t} H_{1}\right. \\
+ & \left.\frac{(-t)^{2}}{2} \int_{0}^{1} d u e^{-(1-u) H_{0} t} H_{1} e^{-u H_{0} t} H_{1}+\ldots\right] .
\end{aligned}
$$

Upon using Eq. (18), Eq. (17) reduces to

$$
\begin{aligned}
\left.\Gamma_{4} \approx \frac{1}{2} \frac{d}{d s}\right|_{0} \frac{1}{\Gamma(s)} \int_{0}^{\infty} d t t^{s} \operatorname{tr} e^{-\left[(p-V)^{2}+m^{2}-\frac{1}{2} F^{\mu \nu} \sigma^{\mu \nu}\right] t}\left[i A_{, \mu}^{\mu}\right. \\
\left.+i \sigma^{\lambda \sigma}\left(2 A^{\lambda} p^{\sigma}+\frac{i}{2} G^{\lambda \sigma}-2 A^{\lambda} V^{\sigma}\right)\right] \gamma^{5} .
\end{aligned}
$$


If $F^{\mu \nu}$ is constant, then by Eqs. (A.1) and (A.2) this becomes

$$
\begin{aligned}
=\left.\frac{1}{2} \frac{d}{d s}\right|_{0} & \frac{1}{\Gamma(s)} \int_{0}^{\infty} d t t^{s} \operatorname{tr} e^{\left[(p-V)^{2}+m^{2}\right] t}\left[\left(\cosh K_{-}\right) P_{+}+\left(\cosh K_{+}\right) P_{-}\right. \\
& \left.+\left(\frac{\sinh K_{-}}{K_{-}} P_{+}+\frac{\sinh K_{+}}{K_{+}} P_{-}\right) w^{\mu \nu} \sigma^{\mu \nu}\right] \\
& {\left[i A_{, \lambda}^{\lambda}+i \sigma^{\lambda \sigma}\left(2 A^{\lambda} p^{\sigma}+\frac{i}{2} G^{\lambda \sigma}-2 A^{\lambda} V^{\sigma}\right)\right] \gamma_{5} }
\end{aligned}
$$

where $w^{\mu \nu}=\frac{1}{2} F^{\mu \nu} t$ and $K_{ \pm}^{2}=2\left(w^{\alpha \beta} w^{\alpha \beta} \pm w^{* \alpha \beta} w^{\alpha \beta}\right)$.

Evaluating the $\gamma$-matrix traces in Eq. (201) leads to

$$
\begin{aligned}
=\frac{d}{d s} & \left.\right|_{0} \frac{i}{\Gamma(s)} \int_{0}^{\infty} d t t^{s} \operatorname{tr} e^{-\left[(p-V)^{2}+m^{2}\right] t}\left\{\left(\cosh K_{-}-\cosh K_{+}\right) A_{, \lambda}^{\lambda}\right. \\
& +2\left[\left(\frac{\sinh K_{-}}{K_{-}}-\frac{\sinh K_{+}}{K_{+}}\right) w^{\lambda \sigma}-2\left(\frac{\sinh K_{-}}{K_{-}}+\frac{\sinh K_{+}}{K_{+}}\right) w^{* \lambda \sigma}\right] \\
& {\left.\left[2 A^{\lambda} p^{\sigma}+\frac{i}{2} G^{\lambda \sigma}-2 A^{\lambda} V^{\sigma}\right]\right\} . }
\end{aligned}
$$

When $V^{\mu}=-\frac{1}{2} F^{\mu \nu} x^{\nu}$, then the result of Schwinger [1]

$$
\begin{aligned}
\left\langle x\left|e^{-(p-V)^{2} t}\right| y\right\rangle & =\frac{i}{(4 \pi t)^{2}} \exp \left(i \int_{y}^{x} d z \cdot V(z)\right) e^{-L(t)} \\
& \exp \left(-\frac{1}{4}(x-y) \cdot F \cdot \cot (F t) \cdot(x-y)\right)
\end{aligned}
$$

can be used to compute the functional trace in Eq. (21). (Here we have $L(t)=$ $\frac{1}{2} \operatorname{tr} \ln \left((F t)^{-1} \sin (F t)\right)$.) In particular, it follows from Eq. (22) that

$$
\begin{aligned}
\operatorname{tr} e^{-(p-V)^{2} t} A^{\lambda} p^{\sigma}= & \operatorname{tr} \int d z\left\langle x\left|e^{-(p-V)^{2} t}\right| z\right\rangle i \partial_{y}^{\sigma}\left\langle z\left|A^{\sigma}\right| y\right\rangle \\
= & \int d x \int d y \delta(x-y) i \partial_{y}^{\sigma}\left[\frac{i}{(4 \pi t)^{2}} \exp \left(i \int_{y}^{x} d z V(z)\right) e^{-L(t)}\right. \\
& \left.\quad \exp \left(-\frac{1}{4}(x-y) \cdot F \cdot \cot (F t) \cdot(x-y)\right) A^{\lambda}(y)\right] \\
= & \frac{i}{(4 \pi t)^{2}} e^{-L(t)} \int d x\left[V^{\sigma}(x) A^{\lambda}(x)+i \partial_{x}^{\sigma} A^{\lambda}(x)\right] .
\end{aligned}
$$

Substitution Eqs. (221) and (231) into Eq. (21) leads to

$$
\begin{aligned}
\Gamma_{4} & \left.\approx \frac{-1}{(4 \pi)^{2}} \frac{d}{d s}\right|_{0} \frac{1}{\Gamma(s)} \int_{0}^{\infty} d t t^{s-2} e^{-L(t)-m^{2} t} \int d x\left\{\left(\cosh K_{-}-\cosh K_{+}\right) A^{\mu, \mu}(x)\right. \\
& \left.-\frac{i}{2} G^{\lambda \sigma}(x) t\left[\left(\frac{\sinh K_{-}}{K_{-}}-\frac{\sinh K_{+}}{K_{+}}\right) F^{\lambda \sigma}-\left(\frac{\sinh K_{-}}{K_{-}}+\frac{\sinh K_{+}}{K_{+}}\right) F^{* \lambda \sigma}\right]\right\} .
\end{aligned}
$$


Expanding Eq. (24) to lowest order in $F^{\lambda \sigma}$ results in

$$
\begin{aligned}
\Gamma_{4} \approx \frac{1}{(4 \pi)^{2}} & \left.\frac{d}{d s}\right|_{0} \frac{1}{\Gamma(s)} \int_{0}^{\infty} d t t^{s-2} e^{-m^{2} t} \int d x\left[\frac{1}{2} t^{2} F^{\lambda \sigma} F^{* \lambda \sigma} A^{\mu, \mu}(x)\right. \\
& \left.-i t G^{\lambda \sigma}(x) F^{* \lambda \sigma}\right] \\
& =\frac{1}{(4 \pi)^{2}} \int d x\left[\frac{1}{m^{2}} F^{\lambda \sigma} F^{* \lambda \sigma} A^{\mu, \mu}+i\left(\ln m^{2}\right) G^{\lambda \sigma} F^{* \lambda \sigma}\right]
\end{aligned}
$$

Neither term in Eq. (25) would arise from the calculation of one-loop Feynman diagrams with plane wave external fields. For $F_{\mu \nu}$ being a constant field, the first term in Eq. (25) is a total derivative. When either $F$ or $G$ (or both) are non-constant the second term is also a total derivative.

\section{THE TWO-DIMENSIONAL LIMIT}

The two dimensional limit of massive electrodynamics has been considered in refs. [22, 23]. If there is an axial coupling between the spinor and an external axial field, this leads to the one-loop effective action

$$
\Gamma_{2}=\ln \operatorname{det}\left(\not p-\not A \sigma^{3}-m\right) \quad(p \equiv-i \partial) .
$$

However, as $\gamma^{\mu} \sigma^{3}=\epsilon^{\mu \nu} \gamma_{\nu}$, this becomes

$$
\Gamma_{2}=\ln \operatorname{det}\left(\not p-A_{\mu} \epsilon^{\mu \nu} \gamma_{\nu}-m\right) .
$$

Consequently, if the background field $A_{\mu}$ corresponds to a constant field strength $A_{\mu}=$ $-\frac{1}{2} F_{\mu \nu} x^{\nu}=-\frac{f}{2} \epsilon_{\mu \nu} x^{\nu}$, then Eq. (27) reduces to

$$
\Gamma_{2}=\ln \operatorname{det}\left(\not p-\frac{f}{2} \not x-m\right)
$$

which is what would be obtained if there were a parity conserving coupling with an external vector field $V_{\mu}=\frac{1}{4} f \partial_{\mu}\left(x^{2}\right)$ which corresponds to a pure gauge field. This effective action should thus be independent of $f$, which we will show explicitly by using Schwinger's technique [1].

If now

$$
\Pi_{\mu}=p_{\mu}-\frac{f}{2} x_{\mu}
$$

then Eq. (28) becomes

$$
\Gamma_{2}=\ln \operatorname{det}^{1 / 2}(\not \Pi+m)(\not \Lambda-m)=\frac{1}{2} \ln \operatorname{det}\left(\Pi^{2}-m^{2}\right)
$$

upon using the two dimensional analogue of Eq. (8) and

$$
\left[\Pi_{\mu}, \Pi_{\nu}\right]=0 .
$$


Regulating $\Gamma_{2}$ using the $\zeta$-function [24, 25] we have

$$
\Gamma_{2}=-\left.\frac{1}{2} \frac{d}{d s}\right|_{0} \frac{1}{\Gamma(s)} \operatorname{tr} \int_{0}^{\infty} d i t(i t)^{s-1} e^{i\left(m^{2}-\Pi^{2}\right) t} .
$$

To evaluate the functional trace in Eq. (32), we use the Hamiltonian approach of ref. [1], defining

$$
\langle x(t) \mid y(0)\rangle=\left\langle x\left|e^{-i H t}\right| y\right\rangle
$$

with

$$
H=-\Pi^{2} .
$$

The equations

$$
\begin{aligned}
i \frac{\partial \Pi^{\mu}(t)}{\partial t} & =\left[\Pi^{\mu}(t), H\right] \\
i \frac{\partial x^{\mu}}{\partial t} & =\left[x^{\mu}(t), H\right]
\end{aligned}
$$

can be integrated to give

$$
\begin{aligned}
\Pi^{\mu}(t) & =\Pi^{\mu}(0) \\
x^{\mu}(t) & =-2 \Pi_{\mu}(0) .
\end{aligned}
$$

Since Eq. (36) is identical to the equations that arise if $f=0$, we see that the effective action in two dimensions for a spinor in the presence of a constant background axial field is just that of a free field.

\section{CONCLUSIONS}

We thus see that the one-loop effective action for a spinor in the presence of a constant background chiral field is closely related to that of considered in refs. [1, 6 6 ] provided $m^{2}=0$. The case in which $m^{2} \neq 0$ in four dimensions has not as yet been given in closed form. Higher order calculations, or those involving non-constant background fields are currently being considered, as is that all-orders approach in the presence of a weak background field [26, 27].

We note the use of projection operators in conjunction with background gauge fields in ref. [28].

\section{Acknowledgments}

We would like to thank Christian Schubert for helpful discussions. Also, Roger Macleod had a helpful suggestion. We appreciate the valuable remarks of the anonymous referee. F. T. Brandt would like to thank CNPq for financial support and the hospitality of the Department of Applied Mathematics of the University of Western Ontario, Canada.

\section{Appendix}

In four dimensional Euclidean space we have the conventions

$$
\left\{\gamma^{\mu}, \gamma^{\nu}\right\}=2 \delta^{\mu \nu}, \quad\left[\gamma^{\mu}, \gamma^{\nu}\right]=2 i \sigma^{\mu \nu}
$$




$$
\begin{gathered}
{\left[\sigma^{\mu \nu}, \sigma^{\lambda \sigma}\right]=2 i\left(\delta^{\mu \lambda} \sigma^{\nu \sigma}-\delta^{\mu \sigma} \sigma^{\nu \lambda}+\delta^{\nu \sigma} \sigma^{\mu \lambda}-\delta^{\nu \lambda} \sigma^{\mu \sigma}\right)} \\
\left\{\sigma^{\mu \nu}, \sigma^{\lambda \sigma}\right\}=2\left(\delta^{\mu \lambda} \delta^{\nu \sigma}-\delta^{\mu \sigma} \delta^{\nu \lambda}\right)-2 \epsilon^{\mu \nu \lambda \sigma} \gamma^{5} \\
\gamma^{\alpha} \gamma^{\beta} \gamma^{\lambda}=\delta^{\alpha \beta} \gamma^{\lambda}-\delta^{\alpha \lambda} \gamma^{\beta}+\delta^{\beta \lambda} \gamma^{\alpha}-\epsilon^{\alpha \beta \lambda \rho} \gamma^{\rho} \gamma^{5} \\
\epsilon^{1234}=1, \quad \gamma^{5}=\gamma^{1} \gamma^{2} \gamma^{3} \gamma^{4}, \quad \operatorname{tr} \gamma^{5}=0 \\
P_{ \pm}=\frac{1 \pm \gamma^{5}}{2} \quad,\left(P_{ \pm}\right)^{2}=P_{ \pm} \quad, \quad P_{ \pm} P_{\mp}=0 \\
P_{ \pm} \gamma^{\mu \nu}=\gamma^{\mu} P_{\mp} \quad, \quad \epsilon_{ \pm} \gamma^{5 \nu \lambda \sigma} \sigma^{\lambda \sigma} .
\end{gathered}
$$

These show that if

$$
e^{\lambda w^{\mu \nu} \sigma^{\mu \nu}}=\left(A_{+}(\lambda) P_{+}+A_{-}(\lambda) P_{-}\right)+\left(B_{+}(\lambda) P_{+}+B_{-}(\lambda) P_{-}\right) w^{\mu \nu} \sigma^{\mu \nu}
$$

then the differential equation

$$
\frac{d}{d \lambda} e^{\lambda w^{\mu \nu} \sigma^{\mu \nu}}=w^{\mu \nu} \sigma^{\mu \nu} e^{\lambda \sigma^{\mu \nu} w^{\mu \nu}}
$$

leads to

$$
\dot{A}_{ \pm}=K_{\mp}^{2} B_{ \pm}, \quad \dot{B}_{ \pm}=A_{ \pm} \quad\left(A_{ \pm}(0)=1, B_{ \pm}(0)=0\right)
$$

where $K_{ \pm}^{2}=2\left(w^{\mu \nu} w^{\mu \nu} \pm w^{\mu \nu} w^{* \mu \nu}\right)$ and $w^{* \mu \nu}=\frac{1}{2} \epsilon^{\mu \nu \lambda \sigma} w^{\lambda \sigma}$. These have the solution when $\lambda=1$

$$
A_{ \pm}=\cosh K_{\mp} \quad B_{ \pm}=\frac{\sinh K_{\mp}}{K_{\mp}} .
$$

The "charge conjugation" matrix $C$ satisfies $C^{-1} \gamma^{\mu} C=-\gamma^{\mu T}, C^{-1} \gamma^{5} C=\gamma^{5 T}$.

In two dimensional Minkowski space, we take

$$
\begin{aligned}
& g^{00}=1=-g^{11} \text { and } \gamma^{0}=\sigma^{1}, \gamma^{1}=i \sigma^{2} \quad \text { so that } \\
& \text { if } \epsilon^{01}=1=\epsilon_{10} \text {, then } \gamma^{\mu} \gamma^{\nu}=g^{\mu \nu}-\epsilon^{\mu \nu} \sigma^{3} \text { and } \gamma^{\mu} \sigma^{3}=\epsilon^{\mu \nu} \gamma_{\nu}
\end{aligned}
$$

(where $\sigma^{i}$ is a Pauli spin matrix). 
[1] J. S. Schwinger, Phys. Rev. 82, 664 (1951).

[2] S. L. Adler, Phys. Rev. 177, 2426 (1969).

[3] J. S. Bell and R. Jackiw, Nuovo Cimento. A60, 47 (1969).

[4] D. Macdonald and D. G. C. McKeon, Int. J. of Theor. Phys. 38, 2371 (1999), 10.1023/A:1026679903218.

[5] D. Van Leeuwen and D. G. C. McKeon, Mod. Phys. Lett. A17, 1961 (2002).

[6] W. Heisenberg and H. Euler, Z. Phys. 98, 714 (1936).

[7] V. Weisskopf, Kgl. Danske Vidensk. Salsk. Mat. Fys. Medd. 14, 1 (1936).

[8] G. V. Dunne, (arxiv hep-th 0406216)

[9] A. L. Maroto, Phys. Rev. D59, 063501 (1999).

[10] D. McKeon, Annals Phys. 224, 139 (1993).

[11] F. Dilkes, D. McKeon, and C. Schubert, JHEP 9903, 022 (1999).

[12] C. Cronstrom, Phys. Lett. B90, 267 (1980).

[13] S. Leupold and H. Weigert, Phys. Rev. D54, 7695 (1996).

[14] S. Leupold, (arxiv hep-th 9609222).

[15] V. Gusynin and I. Shovkovy, Can. J. Phys. 74, 282 (1996).

[16] H. Lee, P. Pac, and H. Shin, Phys. Rev. D40, 4202 (1989).

[17] J. Hauknes, Annals Phys. 156, 303 (1984).

[18] S. P. Kim and D. N. Page, Phys. Rev. D65, 105002 (2002).

[19] N. Narozhnyi and A. Nikishov, Yad. Fiz. 11, 1072 (1970).

[20] G. V. Dunne and T. Hall, Phys. Rev. D58, 105022 (1998).

[21] D. McKeon and T. Sherry, Phys. Rev. D35, 3854 (1987).

[22] C. Adam, Annals Phys. 265, 198 (1998).

[23] M. Krasnansky, Int. J. Mod. Phys. A23, 5201 (2008).

[24] A. Salam and J. Strathdee, Nucl. Phys. B90, 203 (1975).

[25] S. Hawking, Commun. Math. Phys. 55, 133 (1977).

[26] I. K. Affleck, O. Alvarez, and N. S. Manton, Nucl. Phys. B197, 509 (1982).

[27] G. V. Dunne and C. Schubert, Phys. Rev. D72, 105004 (2005).

[28] J. Hur, C. Lee and H. Min, Phys. Rev. D82, 085002 (2010). 